\title{
Lettere di Livia Veneziani a Valery Larbaud, mediatore intellettuale nella fortuna postuma di Italo Svevo
}

\section{Maria Chiara Morighi}

\section{(2) OpenEdition}

Journals

\section{Edizione digitale}

URL: http://journals.openedition.org/studifrancesi/1201

DOI: 10.4000/studifrancesi. 1201

ISSN: 2421-5856

\section{Editore}

Rosenberg \& Sellier

\section{Edizione cartacea}

Data di pubblicazione: 1 décembre 2015

Paginazione: 510-514

ISSN: 0039-2944

\section{Notizia bibliografica digitale}

Maria Chiara Morighi, «Lettere di Livia Veneziani a Valery Larbaud, mediatore intellettuale nella fortuna postuma di Italo Svevo», Studi Francesi [Online], 177 (LIX | III) | 2015, online dal 01 décembre 2016, consultato il 06 janvier 2021. URL: http://journals.openedition.org/studifrancesi/1201 ; DOI: https:// doi.org/10.4000/studifrancesi. 1201

\section{(c)}

Studi Francesi è distribuita con Licenza Creative Commons Attribuzione - Non commerciale - Non opere derivate 4.0 Internazionale. 


\title{
Lettere di Livia Veneziani a Valery Larbaud, mediatore intellettuale nella fortuna postuma di Italo Svevo
}

\begin{abstract}
This article is a commentary on three unpublished letters from the "Valery Larbaud" Multimedia Library of Vichy. The letters, reported here in their entirety, were written to Valery Larbaud by Livia Veneziani, Italo Svevo's widow. In these letters, Mrs. Veneziani asks the French writer to convince James Joyce to write a preface in English for a new edition of the novel Senilità. At the moment of Svevo's death, Joyce had promised Livia his unconditional support in promoting her husband's works; but in time, he seemed to have gone back on his word. Thus, Larbaud remains Livia's only hope in contacting Joyce. Although the friendship between Svevo and Joyce is widely renowned and many studies frequently focus on how it started and grew, little is known about its evolution. It is a fact that Joyce, above all, was responsible for "discovering Svevo" and was one of his most devoted admirers. However, these letters provide a more complete view of their relationship: they prove the existence of some kind of tension between the two writers.
\end{abstract}

I documenti riportati costituiscono parte del materiale, segnalatomi come inedito, da me reperito presso la Mediathèque Valery-Larbaud della città di Vichy.

Si tratta di lettere inviate da Livia Veneziani a Valery Larbaud, testimonianze decisamente interessanti che vanno a completare il profilo della vicenda relativa alla fortuna di Italo Svevo. Il rilievo di tali missive (risalenti rispettivamente al 7 febbraio, 10 marzo e 14 aprile 1931) è dato dal fatto che esse illuminano il rapporto che intercorreva tra James Joyce e lo scrittore triestino. La vulgata racconta che i due intellettuali si incontrarono a Trieste e che, da un iniziale rapporto lavorativo, la loro divenne un relazione di profonda amicizia e reciproca stima. Le lettere in questione non smentiscono tale verità, tuttavia ne ridimensionano la portata. Joyce è una figura legata principalmente all'esordio di Svevo ma cosa avvenne dopo la morte dello scrittore è spesso taciuto; il materiale qui presentato contribuisce a far luce sulla vicenda.

Livia Veneziani, dopo la scomparsa del marito, continua l'opera di diffusione dei romanzi sveviani. Valery Larbaud non perde, nel corso degli anni, il prezioso ruolo di intermediario che aveva ricoperto agli albori della "scoperta" di Italo Svevo. Era stato proprio l'italianisant d'Oltralpe (su suggerimento dello stesso Joyce) a riconoscere la grandezza di Senilità e la deflagrante modernità de La coscienza di Zeno, all'epoca - siamo nel 1925 - quasi completamente trascurati dalla critica italiana. Dopo la lettura di quest'ultimo romanzo Joyce si era permesso di consigliare all'amico triestino qualche strategia promozionale, con una lettera datata 30 gennaio 1924:

Grazie del romanzo con la dedica. Ne ho due esemplari [...]. Sto leggendolo con molto piacere. Perché si dispera? Deve sapere che è di gran lunga il Suo migliore libro. Quanto alla critica italiana non so. Mi faccia mandare degli esemplari di stampa a 
1) M. Valery Larbaud

Chez «Nouvelle Revue Française»

3 rue de Grenelle

2) M. Benjamin Crémieux

Chez «Revue de France»

(troverà l'indirizzo su un esemplare)

3) Mr. T.S. Eliot

Editor

«Criterion»

9 Clarence Gate Mansion

Upper Baker Street

London

4) M. F. Ford

«The Transatlantic Review»

27 Quai d'Anjou

Paris

Parlerò o scriverò in proposito con questi letterati $[\ldots]^{1}$.

La promessa è mantenuta, come dimostra una missiva dello stesso Joyce di qualche tempo successiva: «Caro amico: Mandi i libri senz'altro. Ho già parlato di lei a Larbaud ed a Crémieux $[\ldots]$. Il suo libro sarà certo apprezzato $[\ldots]^{2} \gg$.

Così di fatto avviene, perché lo scrittore irlandese può comunicare quanto segue il primo aprile 1924: «Caro amico: Buone notizie. M. Valery Larbaud ha letto il Suo romanzo. Gli piace molto. Ne scriverà una recensione nella "Nouvelle Revue Française" ${ }^{3}$.

Fu proprio grazie all'acuta sensibilità di Larbaud, unita all'insostituibile contributo di Benjamin Crémieux e di Marie Anne Comnène, che l'opera sveviana ottenne dunque un'inarrestabile risonanza sia in Francia che - se pur tra accese polemiche - in Italia. La graduale ascesa dello scrittore triestino è infatti testimoniata dalla corrispondenza epistolare tra l'autore e i critici francesi che si occuparono della sua opera. Nessuna immagine potrebbe rendere in maniera più efficace l'emozione provata da Svevo nel ricevere la prima missiva di Larbaud:

Ma ecco nel 1925, inatteso, improvviso, un sole di gloria sorgere e illuminare la sua vita. Aveva ormai sessantatre anni. Ricordo quel giorno di gennaio. Eravamo seduti intorno alla grande tavola che riuniva per la colazione assieme a noi anche la famiglia di Letizia con i tre bambini. Egli aprì distrattamente una lettera giunta da Parigi. Cominciò a leggerla ad alta voce e già nell'intestazione restò senza fiato. Cominciava così: «Egregio signore e Maestro». Era la lettera di lode e compiacimento di Valery Larbaud ${ }^{4}$.

La lettera cui Livia Veneziani fa riferimento è quella che apre la corrispondenza tra Larbaud e Svevo e che risale all'11 gennaio 1925. Sin da subito l'intellettuale francese aveva riconosciuto il valore dei romanzi sveviani e si era mostrato disponibile a

(1) Carteggio con James Joyce, Valery Larbaud, Benjamin Crémieux, Marie Anne Comnène, Eugenio Montale, Valerio Jahier, a cura di B. MAIER, dall'Oglio, Milano, 1965, p. 29.

(2) Ivi, p. 31
(3) Ivi, p. 33.

(4) L. Veneziani, Vita di mio marito, stesura di L. Galli, prefazione di E. Montale, Milano, dall'Oglio Editore, 1976, p. 100. Miei i corsivi. 
farsi carico della diffusione degli stessi in una Francia pronta ad accogliere come uno dei suoi figli uno scrittore negletto nella propria patria. La lettura delle opere di Svevo, effettuata dagli intellettuali d'Oltralpe su suggerimento dello scrittore irlandese, ha costituito dunque l'indispensabile premessa per il loro intervento a favore dell'autore, fino ad allora pressocché ignorato. È pertanto evidente che l'intercessione di James Joyce ha rappresentato una tappa imprescindibile nella complicata vicenda del successo editoriale di Svevo.

A quanto risulta dalle testimonianze Joyce, dopo la morte dell'amico, aveva garantito alla signora Veneziani il proprio appoggio e la propria disponibilità ad intervenire, con qualsiasi provvedimento si fosse rivelato necessario, a favore di questi. Quando a Livia viene suggerito di pubblicare in America Senilità corredandola di una prefazione scritta da Joyce (per garantire al romanzo un maggior successo), la signora Veneziani si rivolge allo scrittore irlandese, reclamando quanto le era stato promesso. La risposta di Joyce è un netto rifiuto. Proprio per questo motivo Livia scrive a Larbaud chiedendogli una mediazione, sollecitandolo a convincere lo scrittore irlandese a compiere quest'ultimo passo volto ad onorare la memoria del marito; il tutto, senza esito positivo.

Difficile immaginare una situazione di tal genere, dal momento che sembra incomprensibile il comportamento di Joyce alla luce di quello che si è sempre voluto rappresentare come un rapporto d'amicizia sincera. Eppure segnali di un ripensamento erano già riscontrabili nel 1929, anno in cui esce il numero di «Solaria» (marzo-aprile) dedicato a Svevo, al quale furono chiamati a contribuire, ognuno con il proprio intervento, molti intellettuali che ne avevano riconosciute le capacità artistiche. L'adesione di Joyce all'iniziativa è prettamente formale: dalla lettera che si limita ad inviare da Parigi egli conferma l'amicizia che lo aveva legato allo scrittore, sottolinea il ruolo avuto nella "scoperta di Svevo" ma non dedica una sola parola all'opera dell'amico, demandando la formulazione di giudizi ai critici italiani.

Anche nella comunicazione strettamente privata Joyce conferma la propria renitenza: al fratello Stanislaus egli confessa il rifiuto di scrivere quella prefazione tanto insistentemente richiesta dalla signora Veneziani e lascia trapelare l'esistenza di un'«antica frustrazione»s', sperimentata sin dagli anni della giovinezza, rispetto alla famiglia Schmitz-Veneziani. Joyce avrebbe molto sofferto la situazione di subalternità sociale in cui egli versava: l'evidente distanza di classe tra le due famiglie sarebbe stata - agli occhi di Joyce - costantemente rimarcata dall'entourage dello scrittore triestino, traducendosi nell'esclusione da qualsiasi iniziativa in cui fosse coinvolta l'alta società cittadina, nonché in altri atteggiamenti a questo affini. Che tale senso d'inferiorità fosse o meno legittimato da reali episodi di discriminazione, resta il fatto che Joyce lo percepiva come tale e mai, come dimostrano le circostanze, venne superato; il tutto nonostante Svevo fosse in più di un'occasione intervenuto economicamente a favore dell'amico.

È possibile che tale situazione abbia in qualche modo influenzato la scelta dello scrittore irlandese di non adempiere alla propria promessa di collaborazione.

La prefazione sarà scritta da Stanislaus Joyce e soltanto riletta, senza l'apporto di alcuna modifica, dall'autore dell'Ulisse.

Vani, dunque, i tentativi di Livia di ottenere da questi l'ultimo benevolo gesto nei confronti del marito da poco scomparso.

MARIA CHIARA MORIGHI

(5) G. FinocChiaro ChimirRi, Italo Svevo e James Joyce. Mappa di un incontro memorabile, in Italo
Svevo scrittore europeo, Firenze, Olschki, 1994, p. 61 . 


\section{LIVIA SCHMITZ-SVEVO \\ VILLA VENEZIANI \\ TRIESTE 10}

Monsieur Valery Larbaud

Ce 7 février 1931

Paris

Lors de mon dernier séjour à Paris Joyce m'a montré une lettre qu'il avait reçue de vous de Rome dans laquelle vous lui parliez de mon mari disant que la valeur de son œuvre commence à être reconnue en Italie. Cela m'encourage à m'adresser à vous à propos de la publication de Sénilité en anglais. Zeno a été publié en Angleterre et en Amérique et le succès a été grand d'après les critiques qui me sont parvenues des divers argus de la presse. Mais à ce qu'il paraît le succès de vente en Amérique n'a pas été bien fort car l'éditeur m'écrit: the public did not support Zeno very well. Maintenant Sénilité va être publiée et l'éditeur américain voudrait pouvoir la lancer mieux avec une préface de Joyce. Il se promet un succès immédiat sous l'égide de Joyce. Mais Joyce refuse de faire cette préface disant qu'il ne veut pas déroger à une proposition qu'il s'est fait $[$ sic $]$ de ne jamais écrire de préface ni de $\langle\ldots\rangle$ des lectures. Il dit en outre que son nom "would damn the book in the eyes of readers in England and America for whom I am still a pariah". Je ne suis pas de son avis, ni surtout mon éditeur américain qui insiste et voudrait que j'aille à Paris tâcher de convaincre Joyce. J'ai écrit à Joyce lui rappelant l'offre qu'il m'a fait [sic] lors de la mort de mon mari: "Please remember me if at anytime my help can serve to keep alive a memory of an old friend for whom I had always affection and esteem". J'ai pensé que le moment était venu pour lui d'accomplir cette promesse et j'ai eu un refus. Or je viens vous prier si vous pouvez m'aider à obtenir cette petite faveur. Il n'est pas nécessaire d'écrire des pages. Il me suffirait quelques lignes dans lesquelles il parlerait de l'admiration qu'il a toujours eue pour Senilità; il en savait même quelque passage par cœur. Je me remets à vous et vous serais extrêmement reconnaissante si vous pouviez m'obtenir cela. Ce ne serait qu'accroître ma reconnaissance car si vous saviez quels moments heureux vous avez fait passer à mon mari quand il a reçu votre première lettre et quand il vous a connu à Paris!

Si vous êtes d'opinion qu'il ne faut pas insister auprès de Joyce veuillez me l'écrire et me dire ce que vous pensez. Je vous prie d'excuser le dérangement que je vous cause et agréer, Monsieur, avec mes remerciements mes plus cordiales salutations

Livia Schmitz Svevo

\section{LIVIA SCHMITZ-SVEVO \\ VILLA VENEZIANI TRIESTE 10}

Ce 10 mars 1931

Cher Monsieur Larbaud,

Je viens de recevoir votre carte $\mathrm{du}\langle\ldots\rangle<\ldots>$ et vous remercie vivement. Je regrette de vous savoir souffrant et aussi de vous avoir dérangé dans ces circonstances. Je vois que votre lettre est listée à grand deuil et sans connaître la perte que vous avez subie je vous envoie mes condoléances. J'espère que Joyce va se décider à écrire cette préface et c'est le dernier moment parce que l'éditeur m'écrit que le livre est prêt. Sénilité va sortir aussi à Paris ces jours-ci. L'article de votre ami Justin O'Brien sur l'œuvre de mon mari est magnifique! Je l'ai fait copier et traduire en italien pour l'insérer dans une petite publication qui va sortir le mois prochain.

Je nous renouvelle, cher M. Larbaud, mes remerciements et vous envoie avec mes cordiales salutations les bons souhaits de complète guérison. 
Cher Monsieur Larbaud,

Je vous remercie de votre lettre du 20 mars et de vouloir bien vous occuper de mon affaire malgré que vous soyez souffrant. J'ai vu M. Crémieux à Milan. Il m’a dit que Joyce lui a parlé de ma demande mais qu'il ne veut pas écrire cette préface pour ne pas interrompre ses travaux. Ceci est une excuse qui ne vaut pas grand-chose parce que ce n'est pas dans l'intention de l'éditeur que la préface occupe plusieurs pages. Il suffirait de quelques lignes qu'il pouvait dicter à sa fille ou à Nino Frank qui s'occupe très volontiers des choses de mon mari. Crémieux m'a donné, je crois, un bon conseil. Il m'a dit de prier miss Beach et Mlle Monnier de préparer la préface et de la faire signer par Joyce un jour qu'ils travailleraient ensemble comme cela arrive souvent. Je ne sais pas si miss Beach acceptera ou saura l'écrire. L'éditeur désire que Joyce confirme que Sénilité lui plaisait beaucoup et qu'il a encouragé mon mari à écrire de nouveau. Joyce voulait que Crémieux écrive cette préface. Mais Crémieux a refusé. Et en effet, tout ce que Crémieux a réussi si bien de faire en France pour l'œuvre de mon mari n'aurait pas le même succès en Amérique. Mais vous, cher Monsieur, ne vous sentiriez vous d'écrire ces quelques lignes en Anglais et les envoyer à miss Beach pour les faire signer? Vous auriez ainsi un double mérite à mes yeux et je vous en serais infiniment reconnaissante. Excusez-moi si j'ose vous déranger et vous demander tant de choses. Mais vous avez tant fait pour faire connaître l'œuvre de mon mari en France que peut-être vous voudrez bien aussi avoir un peu de mérite de la faire mieux connaître en Amérique aussi.

Veuillez agréer, Monsieur, avec mes meilleurs vœux pour votre santé, mes plus cordiales salutations

Livia Schmitz Svevo 